\title{
Cross-sectional observational study of the influence of regular physical exercise on sleep quality and presence of sleep disorders in patients assisted in the basic health units of divinopolis, Brazil - a protocol study
}

\begin{abstract}
Introduction: In recent years interest in and studies on sleep disorders has increased. Research indicates a high prevalence of these in adults, being considered a public health problem. Physical exercise, besides being considered a non-pharmacological intervention to improve sleep quality, is fundamental for the development of quality of life, maintenance of mental health and socialization. The regular practice of physical exercise reduces the appearance of diseases, reducing the demand on public health. Due to the lack of knowledge of some professionals, the same is not much used for sleep. The objective of this study will be to compare the quality of sleep and the presence of sleep disorders in patients who perform regular physical exercise and non-regular physical exercise, assisted in Basic Health Unit (BHU) of Divinópolis.

Methods: This study will be a cross-sectional, observational study conducted by the students of the University of the State of Minas Gerais (UEMG) Physiotherapy course, Divinópolis Unit, with patients recruited from the BHU, from November 2016 to March 2018. These individuals will be assessed for sleep quality, presence of sleep disturbances, excessive daytime sleepiness (EDS), risk for obstructive sleep apnea (OSA) and quality of life, addressed according to the respective questionnaires: Pittsburgh Sleep Quality Index (PSQI), Epworth Sleepiness Scale (ESS), Berlin Questionnaire and SF-36 Quality of Life Questionnaire.
\end{abstract}

Discussion: Physical exercise is considered an alternative for improving sleep quality.
Volume 3 Issue I - 2018

Dayse Aparecida Gambôa Leal,' Karina Nunes Assunção,' Luísa Teixeira Pasqualotto, ${ }^{2}$ Walquíria da Mata Santos, ${ }^{3}$ Cíntia Aparecida Santos, ${ }^{2}$ Jacqueline Alves, ${ }^{1}$ Marcos Eugenio Mattos Rocha Faria,' Layra Fernanda Martins,' Rubia Lima Brandão,' Newton Santos de Faria Júnior ${ }^{4}$ 'Undergraduate, University of the State of Minas Gerais, Divinópolis unit (UEMG), Brazil

${ }^{2}$ Undergraduate, PIBIC/UEMG/FAPEMIG scholarship, Brazil ${ }^{3}$ Undergraduate, PAPq/UEMG scholarship, Brazil ${ }^{4}$ Professor, Department of Physiotherapy, University of the State of Minas Gerais-Divinópolis unit (UEMG), Brazil

Correspondence: Newton Santos de Faria Júnior, Department of Physiotherapy of University of the State of Minas GeraisDivinópolis unit (UEMG), Brazil, Zip Code 35.50I-I70, Email nsdfj@yahoo.com.br

Received: December 25, 2017 | Published: February 16, 2018

Keywords: disorders of excessive sonmnolence, sleep, sleep apnea symdromes

Abbreviations: BHU, basic health unit; BMI, body mass index; EDS, excessive daytime sleepiness; ESS, epworth sleepiness scale; ICSD-3, international classification of sleep disorders- $3^{\text {a }}$ edition; NRPE, non-regular physical exercise; PSQI, pittsburgh sleep quality index; RPE, regular physical exercise; SCF, statement of consent form; SF-36, medical outcomes study 36-item short-form health survey; STROBE, strengthening the reporting of observational studies in epidemiology statement; OSA, obstructive sleep apnea; UEMG, university of the state of minas gerais

\section{Introduction}

Sleep is defined as a healthy and restorative state, naturally regenerative and pleasurable, necessary to recover the physical exhaustion common to human experience, due to the constant state of alertness and energy expenditure. ${ }^{1}$ According to studies, sleep disorders have a high prevalence in adults, ranging from 37.2 to $69.4 \%$ of the population. About $16.6 \%$ of the population aged $\geq 50$ years reported extreme or severe sleep problems. ${ }^{2}$ A study carried out in a city in the state of São Paulo showed the occurrence of the disorders in $46.7 \%$ of the people interviewed, reaching mostly women, elderly people and those with obesity. ${ }^{3}$

The disturbances of the sleep wake cycle are considered a public health problem. ${ }^{2}$ They are usually not detected and treated, as there is an ignorance of the population about it. These sleep disorders may lead to reduced cognitive processing, memory deficit, psychiatric disorders, increased traffic and industrial accidents, irritability, metabolic and endocrine changes, development of cardiovascular diseases, thus increasing the number of hospitalizations and mortality.

Currently, physical exercise is considered a non-pharmacological alternative for improving sleep quality, ${ }^{4}$ favors healthier life habits, reducing the appearance of diseases, thus representing a saving for public health, since it reduces the demand for health posts, health and hospitals. Being of easy application and access to the population, the physical exercise has contributed to the improvement of the sleep and quality of life, however by the lack of knowledge of some professionals the same is not very used for this purpose. The objective of this study will be to compare the quality of sleep and the presence of sleep disorders in patients who perform regular physical exercise (RPE) and non-regular physical exercise (NRPE), assisted in Basic Health Unit (BHU) of Divinópolis. 


\section{Methods}

\section{Study design and ethical consideration}

This study will be an observational, cross-sectional study conducted by the students of the University of the State of Minas Gerais (UEMG) Physiotherapy course, Divinópolis Unit, with patients recruited from BHU in the city of Divinópolis-MG, between November 2016 and March 2018. The study design will follow the norms of the "Strengthening the Reporting of Observational Studies in Epidemiology (STROBE) statement" (Figure 1) $)^{5,6}$ and will agree with the ethical standards set forth in the Declaration of Helsinki 1961 (revised in Hong Kong in 1989 and of Edinburgh in Scotland in 2000) and the Guidelines and Regulatory Norms for research involving human beings of the National Health Council of the Brazilian Ministry of Health, resolution 196/96 updated by 466/2012. The present study was approved by the Research Ethics Committee with Human Subjects of the University of the State of Minas Gerais under protocol number 1,475,521/2016 and authorized by the Municipal Health Office Secretary of Divinópolis. Of all the patients involved will be obtained the Statement of Consent Form (SCF), being allowed the removal at any time without any onus.

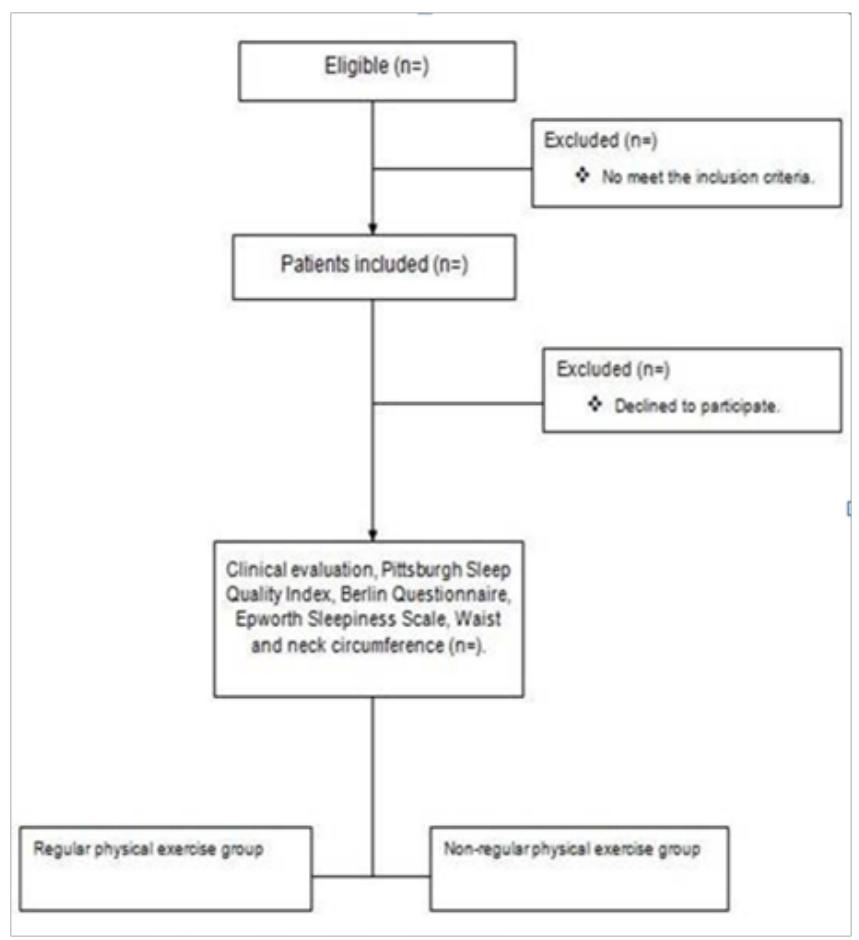

Figure I Flow chart representing the study design.

\section{Subjects}

Participating in this study will be patients assisted in the BHU of the city of Divinópolis-MG, after signing the SCF. This sample will be consecutive and of convenience and stratified as to the sociodemographic variables, comorbidities, presence or not of sleep disorders and other variables pertinent to the study. The patients who participate in the physical exercise groups in the BHUs, perform three days a week, two days lasting 60 minutes, divided into 40 minutes of walking, 10 minutes of stretching and 10 minutes of muscle strengthening, alternating between upper and lower limbs. On the third day, the group does warm-up, stretching and walking for at least 40 minutes without supervision.
The study will include patients with BHUSs in Divinópolis, with a minimum age of 18 years, of both sexes, without cognitive deficit that would interfere in the comprehension to answer the questionnaires. From the RPE group will participate those with at least three months of supervised physical exercise in the BHUs and in the group of NRPE will participate patients from the BHUs selected at random while awaiting care, who do not perform physical exercise. Those patients who do not agree to answer the questionnaires and with comorbidities that may influence the results of the evaluations will be excluded.

\section{Evolution protocol}

\section{Clinical evolution}

The patients will be evaluated in the BHUs according to the specific evaluation form, which contains personal data, objective evaluation of the heart rate, systemic arterial pressure, body mass index (BMI), socioeconomic data, comorbidities, physical examination and habits of daily living. In the RPE group, systemic blood pressure will be measured after the patient is seated at rest for 10 minutes before the beginning of the exercise by the auscultatory method. ${ }^{7}$ In the NRPE group, the systemic arterial pressure will be measured at the time of the evaluation after the patient remains seated at rest for 10 minutes. The weight and height will be evaluated through a scale provided by the BHUs. The BMI calculation is performed by BMI classification of the World Health Organization. ${ }^{8}$ Already the neck circumference will be measured with the individual in the seated position, at the level of the anterior border of the cricoid cartilage, both using a non-elastic tape measure with precision of 1 millimeter. ${ }^{9}$ According to the Brazilian Otorhinolaryngology Society, normal values are $\leq 43.2 \mathrm{~cm}$ for men and $\leq 38 \mathrm{~cm}$ for women. ${ }^{10}$ The abdominal waist circumference will also use a non-elastic tape measure for measurement. It will be measured at the midpoint between the lower margin of the last rib and the iliac crest. ${ }^{8}$ Values $\geq 94 \mathrm{~cm}$ for men and $\geq 80 \mathrm{~cm}$ for women are considered by the $\mathrm{V}$ Brazilian Guidelines on Dyslipidemia and Prevention of Atherosclerosis as a reference for increased cardiovascular risk. ${ }^{11}$

\section{Pittsburgh sleep quality index (PSQI)}

The Portuguese version of the Pittsburgh Sleep Quality Index (PSQI) ${ }^{12,13}$ will be applied to assess sleep quality of patients. The questionnaire contains 19 questions, divided into 7 areas scored separately. The sum of these points (range:0-21) provides an overall measure of sleep quality, with high scores indicating insufficient sleep ( $>5$ is indicative of poor sleep quality). The items evaluated are related to the subjective quality of sleep, duration of sleep, sleep latency, habitual sleep efficiency, sleep disorders, use of sleep-related medications, and sleep impact on daytime dysfunctions.

\section{Epworth sleepiness scale and berlin questionnaire}

Two specific sleep questionnaires will also be applied. In order to determine the presence of EDS, the Portuguese version of ESE ${ }^{14}$ ${ }^{16}$ will be used. It is a simple and self-applicable questionnaire that addresses situations involving the occurrence of daytime sleepiness during normal daily activities in adults. Participants will be instructed to rate their likelihood of feeling the urge to sleep or doze off in eight situations, on a scale of 0 to 3 .

In order to determine the risk for obstructive sleep apnea (OSA), an individualized clinical questionnaire, called the Berlin Questionnaire, ${ }^{17}$ will be applied. This questionnaire has 10 items organized into three categories as follows: apnea and snoring, daytime somnolence and systemic arterial hypertension and obesity. All positively marked 
responses are considered risk factors for OSA. Patients were classified as high risk or low risk for OSA. A patient is considered at high risk for OSA if two or more of the three categories are positive.

\section{Quality of life}

To evaluate the quality of life, the generic questionnaire SF-36 (Medical Outcomes Study 36-Item Short-Form Health Survey) ${ }^{18}$ validated version for the Portuguese language in Brazil. ${ }^{19}$ The SF-36 is an instrument of easy administration and understanding, but not so extensive. It contains 36 items, of which 35 are grouped into eight dimensions (functional capacity, pain, physical aspects, emotional aspects, social aspects, mental health, vitality and general health status) and a last item that evaluates health change in the time. For each dimension, SF-36 items are coded, grouped, and transformed into a scale from zero (poorer health) to 100 (better health).

\section{Quality control}

The researchers responsible for data acquisition in the study were given specific training for the safety of data quality. Periodic external monitoring is performed to verify the application of the methodology for an information acquisition and different tests.

\section{Statistical analysis}

Numerical data will be known as mean and standard, there are no cases of variables with normal distribution, and median and interquartile range for those with asymmetric distribution. Categorical data will be describe as absolute number and percentage of total.

The Kolmogorov-Smirnov normality test will be performed in order to determine the normality of data. According to the embodiment of the sample stratification, Student's t-test when comparing paired samples. For the comparisons between variables, for the quantitative variables, the Student's t test or the non-parametric Mann-Whitney test will be used. When the variables were qualitative, the Chi-square test or Fisher's exact test will be used, depending on the case. The statistical analysis will be used statistical software GraphPad Prism Software Inc., version 5.01 for Windows 2007 (San Diego, CA, USA) was used. The level of statistical significance is set at $5 \%$ for all testis $(\mathrm{p}<0.05)$.

\section{Discussion}

Physical exercise is considered a non-pharmacological alternative to improve sleep quality, favors healthier life habits, reducing the appearance of diseases, thus representing an economy for public health, since it reduces the demand for health posts and hospitals. Being of easy application and access to the population, the physical exercise has contributed to the improvement of the sleep and quality of life, however by the lack of knowledge of some professionals the same is not very used for this purpose.

\section{Acknowledgements}

LTP and CAS receives a grant from the Fundação de Amparo à Pesquisa do Estado de Minas Gerais (local acronym FAPEMIG PIBIC/UEMG/FAPEMIG). WMS receives grant from the Programa Institucional de Apoio à Pesquisa da UEMG (local acronym PAPq -UEMG). All authors contributed equally to this work.

\section{References}

1. Dement WC. A personal history of sleep disorders medicine. $J$ Clin Neurophysiol. 1990;7(1):17-47.

2. Stranges S, Tigbe W, Gomez OFX, et al. Sleep problems: an emerging global epidemic? Findings from the in depth WHO-SAGE study among more than 40,000 older adults from 8 countries across Africa and Asia. Sleep. 2012;35(8):1173-1181.

3. Zanuto EAC, Lima MCSD, Araújo RGD, et al. Distúrbios do sono em adultos de uma cidade do estado de São paulo. Rev Bras de Epidemiol. $2015 ; 18(1): 42-53$.

4. Pereira EG, Gordia AP, Quadros TMG. Padrão do sono em universitários brasileiros e sua relação com a prática de atividades físicas: uma revisão da literatura. Rev Bras Cienc Saúde. 2011;30(9):55-60.

5. Von EE, Altman DG, Egger M, et al. Strengthening the reporting of observational studies in epidemiology (STROBE) statement: guidelines for reporting observational studies. BMJ. 2007;335(7624):806-808.

6. Malta M, Cardoso LO, Bastos FI, et al. Strobe initiative: guidelines on reporting observational studies. Rev Saúde Pública. 2010;44(3):559-565.

7. Tavares A, Rodrigues CIS, Consolim CF, et al. VI Diretrizes Brasileiras de Hipertensão. ArqBrasCardiol. 2010;95(1):1-51.

8. WHO. Obesity : preventing and managing the global epidemic. Report of a WHO consultation. World health organization, Geneva. 2000;894(i-xii):1-253.

9. Zen V, Fuchs FD, Wainstein MV, et al. Neck circumference and central obesity are independent predictors of coronary artery disease in patients. Am J Cardiovasc Dis. 2012;2(4):323-330.

10. Pinto JÁ, Ballester LMG, Wanderley VPBM, et al. Medidas antropométricas preditoras da gravidade da apneia obstrutiva do sono. Braz j Otorhinolaryngol. 2011;77(4):516-521.

11. Xavier HT, Izar MC, Faria NJR, et al. IV diretriz brasileira sobre dislipidemias e prevenção da aterosclerose. Arq Bras Cardiol. 2013;101(4):1-22.

12. Buysse DJ, Reynolds CF, Monk TH, et al. The pittsburgh sleep quality index: a new instrument for psychiatric practice and research. Psychiatr Res. 1989;28(2):193-213.

13. Bertolazi AN, Fagondes SC, Holf LS, et al. Validation of the brazilian portuguese language of the pittsburgh sleep quality Index. Sleep Med. 2011;12(1):70-75

14. Johns MW. Reliability and factor analysis of the epworth sleepiness scale. Sleep. 1991;14(6):540-545.

15. Bertolazi AN, Fagondes SC, Hoff LS, et al. Portuguese-language version of the epworth sleepiness scale: validation for use in Brazil. J Bras Pneumol. 2009;35(9):877-883.

16. Slater G, Steier J. Excessive daytime sleepiness in sleep disorders. $J$ Thorac Dis. 2012;4(6):608-616.

17. Netzer NC, Stoohs RA, Netzer CM, et al. Using the berlin questionnaire to identify patients at risk for the sleep apnea syndrome. Ann InternMed. 1999;131(7):485-491.

18. Ware JEJr, Sherbourne CD. The MOS 36-item short-form health survey (SF-36). I. Conceptual framework and item selection. Med Care. 1992;30(6):473-483.

19. Ciconelli RM, Ferraz MB, Santos W, et al. Brazilian-portuguese version of the SF-36. A reliable and valid quality of life outcome measure. Rev Bras Reumatol. 1999;39(3):143-150.

\section{Conflict of interest}

The author declares no conflict of interest. 